\title{
PERFIL DE SENSIBILIDADE A ANTIMICROBIANOS DE BACTÉRIAS PATOGÊNICAS HUMANAS ISOLADAS DE LEITE CRU
}

\section{Antimicrobial susceptibility profile of human pathogenic bacteria isolated from raw milk}

\author{
Reginara Teixeira da Silva ${ }^{2}$, Joyce Bitencourt Athayde Lopes ${ }^{2}$, Kleydejany Lima Lemos de \\ Oliveira $^{3}$, José Carlos Ribeiro Júnior ${ }^{4}$, Vanerli Beloti ${ }^{5}$
}

\section{RESUMO}

A presença de bactérias no leite cru, associada ao possível uso indiscriminado de antimicrobianos no gado leiteiro, pode influenciar no surgimento de bactérias resistentes. O perfil de sensibilidade a antimicrobianos de seis cepas bacterianas patogênicas, isoladas de leite cru do estado do Maranhão (Acinetobacter septicus, Hafnia alvei, Lactococcus garvieae, Staphylococcus epidermidis, Pantoea spp. e Klebsiella spp.), foi avaliado neste estudo por meio do teste de antibiograma por difusão de disco em Ágar Müeller Hinton, e para a interpretação das categorias de sensibilidade foram utilizados os critérios previamente estabelecidos. $\mathrm{O}$ antimicrobiano com menor eficiência foi a lincomicina, onde 83,33\% das cepas apresentaram resistência. A gentamicina e a tetraciclina apresentaram 100\% de eficiência frente os isolados deste estudo. Klebsiella spp. destacara-se por apresentar resistência $>50 \%$ aos antimicrobianos testados. No entanto, observouse multirresistência em 50\% dos isolados: Hafnia alvei, Pantoea spp. e Klebsiella spp. Os resultados verificados demonstram a multirresistência de patógenos de

1 Universidade Estadual de Londrina, Programa de Pós-Graduação em Biotecnologia, Rodovia Celso Garcia Cid, PR 445, km 380, 86.057-970, Londrina, PR, Brasil. E-mail: reginara.teixeira@uel.br

2 Instituto Federal de Educação Ciência e Tecnologia do Maranhão, Departamento de Ensino, Caxias, MA, Brasil.

3 Instituto Federal de Educação Ciência e Tecnologia do Maranhão, Departamento de Ensino, São Luís, MA, Brasil.

4 Universidade Federal do Tocantins, Escola de Medicina Veterinária e Zootecnia, Araguaína, TO, Brasil.

5 Universidade Estadual de Londrina, Departamento de Medicina Veterinária Preventiva, Londrina, PR, Brasil.

* Autor para correspondência 
grande importância para a saúde pública, e o leite cru como uma possível fonte de veiculação desses micro-organismos multirresistentes.

Palavras-chave: microbiologia láctea; qualidade de alimentos; bactérias resistentes.

\section{ABSTRACT}

The presence of bacteria in raw milk associated with the possible indiscriminate use of antimicrobials in dairy cattle can influence the emergence of resistant bacteria. The antimicrobial susceptibility profile of nine pathogenic bacterial strains isolated from raw milk from the State of Maranhão (Acinetobacter septicus, Hafnia alvei, Lactococcus garvieae, Staphylococcus epidermidis, Pantoea spp. and Klebsiella spp.) was evaluated in this study employing the test of disc diffusion antibiogram in Müller Hinton Agar, and for the interpretation of the sensitivity categories, it was used the criteria previously established. The least efficient antimicrobial was lincomycin, in which $83.33 \%$ of the strains showed resistance. Gentamicin and tetracycline were $100 \%$ efficient against the isolates of this study. Klebsiella spp. were resistant to more than $50 \%$ of the antimicrobials tested. However, multi-resistance was observed in $50 \%$ of the isolates: Hafnia alvei, Pantoea spp. and Klebsiella spp. The verified results demonstrate the multiresistance of pathogens of great importance for public health, and the raw milk as a possible source of delivery of these multi-resistant microorganisms.

Keywords: milk microbiology; food quality; resistant bacteria.

\section{INTRODUÇÃO}

As bactérias, mesmo quando não são patogênicas para o homem a partir da ingestão de alimentos contaminados, se resistentes a antimicrobianos e presentes em abundância na cadeia alimentar, podem ter um efeito grave nas futuras opções de tratamento para uma ampla gama de infecções causada por estes micro-organismos (OVERDEVEST et al., 2011).

A maioria dos micro-organismos presentes nos alimentos podem ser eliminados facilmente através de tratamentos térmicos. Porém, quando métodos de tratamento não são utilizados ou são ineficientes, os alimentos tornam-se potenciais veiculadores de doenças, como acontece com o leite no Brasil, onde o consumo direto ou de derivados crus é ainda frequente. $\mathrm{O}$ estudo de Pieri et al. (2014) relata que 18,5\% dos brasileiros entrevistados consomem regularmente leite e derivados crus sem qualquer tipo de tratamento térmico ou processo tecnológico que confira segurança alimentar. Dessa forma, o leite pode ser a fonte de contaminação aos consumidores de micro-organismos patogênicos e/ou resistentes aos antimicrobianos.

A resistência dos seres procarióticos aos antimicrobianos tem origem genética e está relacionada com a codificação de diferentes mecanismos bioquímicos, realizada por determinados genes que inibem a ação das drogas. Essa resistência é originada na maioria das vezes por mutações que ocorrem na sequência de bases do material genético, resultando em cópias do DNA com erros sequenciais que permitem a multiplicação mesmo na presença do antibiótico (FRACALANZZA, 2007), selecionando cepas resistentes. 
Embora a resistência bacteriana seja um fenômeno natural e intrínseco aos antimicrobianos desde sua descoberta por Alexander Fleming, em 1928, de acordo com a literatura especializada, de maneira geral, o uso indiscriminado de antimicrobianos é um dos principais fatores para a geração e a intensificação dessa resistência até os dias atuais (ESTRELA, 2018).

As infecções causadas por essas bactérias resistentes podem ser consideradas como emergentes, pois o seu tratamento é cada vez mais limitado pela multirresistência adquirida por esses patógenos às diferentes classes de antimicrobianos (SANTOS, 2004; ROCHA et al., 2015). Dessa forma, novos antimicrobianos estão sendo desenvolvidos. Nos últimos cinco anos o Food and Drug Administration (FDA, 2019) aprovou o fosfato de tedizolida (2014), oritavancina (2014), dalbavancina (2014), ceftolozano + tazobactam (2014), ceftazidima + avibactam (2015), bezlotoxumab (2016), delafloxacina (2017), meropem + vaborbactam (2017), omadaciclina (2018) e imipenem + cilastatina + relebactam (2019).

O uso de medicamentos veterinários para controlar, tratar e/ou prevenir as enfermidades do gado leiteiro, quando realizado de forma incorreta, pode deixar resíduos no leite e, consequentemente, nos derivados lácteos (ALMEIDA et al., 2003), podendo causar também essa resistência bacteriana com repercussões na qualidade do leite para consumo (SILVA; FAGNANI, 2015).

Considerando a potencial resistência antimicrobiana de micro-organismos do leite e o risco ao consumo de produtos lácteos contaminados por micro-organismos resistentes, o presente trabalho teve por objetivo verificar a suscetibilidade antimicrobiana de cepas de micro-organismos isolados do leite descritas como causadores de infecções clínicas e hospitalares.

\section{MATERIAL E MÉTODOS}

\section{Amostragem}

Foram selecionadas seis cepas bacterianas, isoladas de leite cru de regiões do estado do Maranhão, por tratarem-se de bactérias que não apresentam relação com doenças veiculadas por alimentos, porém, são conhecidas por envolvimento em infecções diversas. As bactérias estudadas e sua implicação em saúde pública estão relacionadas na Tabela 1 .

\section{Teste de antibiograma}

As cepas bacterianas foram testadas por meio da técnica de antibiograma segundo as normas do Clinical and Laboratory Standards Institute (CLSI, 2012). Previamente, as cepas bacterianas foram replicadas em $2 \mathrm{~mL}$ de caldo infusão de cérebro e coração (BHI) (Acumedia, EUA) e incubadas a $37{ }^{\circ} \mathrm{C}$ até atingir turvação correspondente ao tubo 0,5 da escala de Mac Farland, que corresponde a, aproximadamente, $1,5 \times 10^{8} \mathrm{UFC} / \mathrm{mL}$. Em seguida, alíquotas dos inóculos foram diluídas em solução salina $0,85 \%$ estéril e submetidas ao teste de sensibilidade in vitro a antimicrobianos, pelo método de difusão de discos em ágar Müeller Hinton (BAUER, et al., 1966).

Foram selecionados antimicrobianos comumente utilizados por pecuaristas das regiões de onde foram isoladas as bactérias estudadas. Sendo eles: $\beta$-lactâmicos (ampicilina $10 \mathrm{mcg}$, amoxicilina $10 \mathrm{mcg}$, penicilina 10 U.I.); aminoglicosídeos (gentamicina $10 \mathrm{mcg}$, estreptomicina 10 $\mathrm{mcg}$ ); polipeptídicos (bacitracina 10 U.I.); fluoroquinolonas (norfloxacina $10 \mathrm{mcg}$ ); tetraciclinas (tetraciclina $30 \mathrm{mcg}$ ); macrolídeos (eritromicina $15 \mathrm{mcg}$ ); sulfonamidas (sulfazotrim $25 \mathrm{mcg}$ ); lincosamidas (lincomicina $2 \mathrm{mcg}$ ) (CECON, São Paulo, Brasil). As leituras dos halos de inibição foram 
realizadas após 24 horas de incubação a $37^{\circ} \mathrm{C}$, sendo medidos em milímetros e os resultados interpretados como sensíveis, intermediários ou resistentes às drogas testadas, de acordo com critérios estabelecidos pelo documento M100-S25 (CLSI, 2015).

\section{RESULTADOS E DISCUSSÃO}

Na Tabela 2 encontram-se os resultados obtidos a partir do teste de antibiograma, observa-se que os antibióticos foram agrupados de acordo com sua classe e classificados como resistente, intermediário ou sensível.

Das seis cepas testadas todas apresentaram sensibilidade a pelo menos cinco tipos de antimicrobianos utilizados. Observouse que para gentamicina e tetraciclina, todas as cepas foram sensíveis (Tabela 2).

Micro-organismos multirresistentes são aqueles resistentes a vários medicamentos antimicrobianos pertencentes a diferentes classes ou subclasses químicas por meio de

Tabela 1 - Cepas bacterianas isoladas de leite cru de regiões do estado do Maranhão por Lima (2016) conhecidas como causadoras de infecções

\begin{tabular}{|c|c|c|}
\hline CEPAS BACTERIANAS & GRAM & Infecções \\
\hline Acinetobacter septicus & Negativo & $\begin{array}{l}\text { Infecções em pacientes graves em unidades de } \\
\text { terapia intensiva (KILIC et al., 2008). }\end{array}$ \\
\hline Hafnia alvei & Negativo & $\begin{array}{l}\text { Infecções sistêmicas, incluindo septicemia } \\
\text { e pneumonia. Infecções intestinais e extra } \\
\text { intestinais (JANDA; ABBOTT, 2006). }\end{array}$ \\
\hline Lactococcus garvieae & Positivo & $\begin{array}{l}\text { Infecção mais comum é a endocardite (WILBRING } \\
\text { et al., 2011), além de ser um importante patógeno } \\
\text { de peixes (VENDRELL et al., 2006). }\end{array}$ \\
\hline Staphylococcus epidermidis & Positivo & $\begin{array}{l}\text { Infecções cardíacas, hospitalares e à mastite } \\
\text { bovina. São bastante adaptadas às glândulas } \\
\text { mamárias das vacas e parecem usá-la como } \\
\text { reservatório de fonte de infecção (PIESSENS et } \\
\text { al., 2012, TOMAZI et al., 2014) }\end{array}$ \\
\hline Pantoea spp. & Negativo & $\begin{array}{l}\text { Infecção de tecidos moles e/ou osso seguidas } \\
\text { de traumas penetrante por vegetação. A espécie } \\
\text { Pantoea agglomerans já foi encontrada em } \\
\text { feridas, sangue e urina, como patógeno oportunista } \\
\text { (BICUDO et al., 2007; POSSUELO; RENNER, } \\
\text { 2014). }\end{array}$ \\
\hline Klebsiella spp. & Negativo & $\begin{array}{l}\text { Isoladas por Oliveira et al. (2011) em uroculturas, } \\
\text { secreções, hemoculturas e pontas de cateter. } \\
\text { Descrita provocando infecções na corrente } \\
\text { sanguínea, respiratória e do trato urinário em } \\
\text { pacientes da UTI (PERNA et al., 2015). }\end{array}$ \\
\hline
\end{tabular}


vários mecanismos (MAGIORAKOS et al., 2012). A cepa de $H$. alvei avaliada apresentou resistência à quatro antimicrobianos: eritromicina, lincomicina, bacitracina e penicilina que pertencem às classes macrolídeos, lincosamidas, polipeptídeos, glicopeptídeos e $\beta$-lactâmicos, respectivamente, conforme Tabela 2. Portanto, pode ser classificada como uma cepa multirresistente.

No entanto, de todas as cepas testadas, a cepa de Klebsiella sp. apresentou um perfil maior de resistência, frente aos antimicrobianos utilizados, sendo esta, sensível apenas na presença de cinco dos doze testados: gentamicina, estreptomicina, norfloxacina, tetraciclina e sulfazotrim, logo, também apresentou perfil de multirresistência.

As bactérias $H$. alvei e Pantoea sp. testadas nesta pesquisa, apresentaram resultados semelhantes, se diferenciando apenas diante da amoxicilina, sendo a primeira resistente e a segunda sensível. Na presença de eritromicina, lincomicina, bacitracina e penicilina mostraram-se resistentes, já para ampicilina, gentamicina, estreptomicina, norfloxacina e tetraciclina foram sensíveis. É importante destacar que os gêneros Klebsiella sp., Hafnia sp. e Pantoea sp. pertencem à mesma família Enterobacteriaceae, e apresentaram um perfil de sensibilidade muito similar diante das diferentes classes de antimicrobianos usadas.

No estudo realizado por Salaberry et al. (2016) que fez uma análise microbiológica e do perfil de sensibilidade do Staphylococcus spp. em mastite subclínica de caprinos leiteiros, ressalta-se que $S$. epidermidis apresentou maior resistência para a amoxicilina e ampicilina, seguida da penicilina e oxacilina, em relação aos demais antimicrobianos testados em seu estudo. Diferentemente, no presente trabalho, a cepa de $S$. epidermidis apresentou sensibilidade a todos os antimicrobianos usados, inclusive a amoxicilina, ampicilina e penicilina.

Com o objetivo de determinar a prevalência de Acinetobacter spp. em amostras de leite em tanque (BTM) de diferentes propriedades da Coreia e analisar a susceptibilidade antimicrobiana,

Tabela 2 - Perfil de sensibilidade das bactérias patogênicas isoladas do leite cru de regiões do estado do Maranhão, interpretados de acordo com o CSLI (2016)

\section{Categorias de antibióticos*}

\begin{tabular}{cccccccccccc}
\cline { 2 - 10 } Cepa & \multicolumn{3}{c}{$\boldsymbol{\beta}$-LACTÂMICOS } & \multicolumn{2}{c}{ AMINO } & POL & FLU & TET & MAC & SUL & LIN \\
\cline { 2 - 11 } & AMP & AMO & PEN & GEN & EST & BAC & NOR & TET & ERI & SUT & LIN \\
\hline A. septicus & $\mathrm{S}$ & $\mathrm{S}$ & $\mathrm{I}$ & $\mathrm{S}$ & $\mathrm{S}$ & $\mathrm{S}$ & $\mathrm{S}$ & $\mathrm{S}$ & $\mathrm{I}$ & $\mathrm{S}$ & $\mathrm{R}$ \\
H. alvei & $\mathrm{S}$ & $\mathrm{R}$ & $\mathrm{R}$ & $\mathrm{S}$ & $\mathrm{S}$ & $\mathrm{R}$ & $\mathrm{S}$ & $\mathrm{S}$ & $\mathrm{R}$ & $\mathrm{S}$ & $\mathrm{R}$ \\
L. garvieae & $\mathrm{S}$ & $\mathrm{S}$ & $\mathrm{S}$ & $\mathrm{S}$ & $\mathrm{R}$ & $\mathrm{S}$ & $\mathrm{I}$ & $\mathrm{S}$ & $\mathrm{S}$ & $\mathrm{R}$ & $\mathrm{R}$ \\
S. epidermidis & $\mathrm{S}$ & $\mathrm{S}$ & $\mathrm{S}$ & $\mathrm{S}$ & $\mathrm{S}$ & $\mathrm{S}$ & $\mathrm{S}$ & $\mathrm{S}$ & $\mathrm{S}$ & $\mathrm{S}$ & $\mathrm{S}$ \\
Pantoea spp. & $\mathrm{S}$ & $\mathrm{S}$ & $\mathrm{R}$ & $\mathrm{S}$ & $\mathrm{S}$ & $\mathrm{R}$ & $\mathrm{S}$ & $\mathrm{S}$ & $\mathrm{R}$ & $\mathrm{S}$ & $\mathrm{R}$ \\
Klebsiella spp. & $\mathrm{R}$ & $\mathrm{R}$ & $\mathrm{R}$ & $\mathrm{S}$ & $\mathrm{S}$ & $\mathrm{R}$ & $\mathrm{S}$ & $\mathrm{S}$ & $\mathrm{R}$ & $\mathrm{S}$ & $\mathrm{R}$
\end{tabular}

* (S) Sensível; (I) Intermediário; (R) Resistente; AMINO - Aminoglicosídeos; POL - Polipeptídicos; FLU Fluoroquinolonas; TET - Tetraciclinas; MAC - Macrolídeos; SUL - SulfonamidaS; LIN - Lincosamidas; AMP - ampicilina; AMO - amoxicilina; PEN - penicilina; GEN - gentamicina; EST - estreptomicina; BAC - bacitracina; NOR - norfloxacina; ERI - eritromicina; SUT - sulfazotrim; LIN - lincomicina. 
Gurung et al. (2013) verificou que os isolados de Acinetobacter spp. foram menos suscetíveis à tetraciclina $(17,6 \%)$, seguidos por trimetoprim-sulfametoxazol $(15,9 \%)$, ceftazidima $(10,8 \%)$ e ampicilinasulbactam (10,2\%). No presente estudo, a cepa de Acinetobacter septicus apresentou sensibilidade a todos os antimicrobianos testados, sendo resistente apenas na presença de lincosamidas e um perfil intermediário diante de penicilina e eritromicina.

A multirresistência em bactérias patogênicas apresenta muitas consequências para o homem, tais como: maior tempo de internação hospitalar, redução da qualidade de vida, maior probabilidade de morte devido ao tratamento inadequado ou atrasado, aumento da cobertura de seguro particular, custos adicionais para hospitais, dentre outros (CANTAS et al., 2013).

Além disso, como em regiões do interior do Maranhão, onde é costume a produção de derivados lácteos crus e informais/não inspecionados, é possível que esses micro- organismos prevaleçam nos derivados informalmente produzidos a partir das amostras de leite das quais foram isolados.

Considerando somente a efetividade dos princípios ativos avaliados pelo presente trabalho, é possível observar na Tabela 3 o percentual de eficácia dos antimicrobianos frente as cepas testadas.

Foi possível observar que os antimicrobianos gentamicina e tetraciclina apresentaram $100 \%$ de eficácia no combate às seis cepas testadas. Observou-se que para ampicilina, amoxilina, bacitracina, estreptomicina, norfloxacina e sulfazotrim houve um percentual de eficiência $\geq 50 \%$. Os antimicrobianos que apresentaram melhores resultados no presente estudo foram a gentamicina e tetraciclina, pois, todas as cepas testadas apresentaram sensibilidade, conforme Tabela 3. É possível observar, ainda, que o antimicrobiano com menor eficiência frente as cepas testadas neste trabalho foi a lincomicina, apresentando o percentual de $83,33 \%$ de cepas resistentes.

Tabela 3 - Percentual de eficácia dos antimicrobianos testados em bactérias patogênicas isoladas de leite cru de regiões do estado do Maranhão

\begin{tabular}{lccc}
\hline \multicolumn{1}{c}{ Antimicrobianos } & Sensível & Intermediária & Resistente \\
\hline Ampicilina & $83,33 \%$ & $0,00 \%$ & $16,67 \%$ \\
Amoxilina & $66,67 \%$ & $0,00 \%$ & $33,33 \%$ \\
Bacitracina & $50,00 \%$ & $0,00 \%$ & $50,00 \%$ \\
Eritromicina & $33,33 \%$ & $16,67 \%$ & $50,00 \%$ \\
Estreptomicina & $83,33 \%$ & $11,11 \%$ & $16,67 \%$ \\
Gentamicina & $100,0 \%$ & $0,00 \%$ & $0,00 \%$ \\
Lincomicina & $16,67 \%$ & $0,00 \%$ & $83,33 \%$ \\
Norfloxacina & $83,33 \%$ & $16,67 \%$ & $0,00 \%$ \\
Penicilina & $33,33 \%$ & $0,00 \%$ & $50,00 \%$ \\
Sulfazotrim & $83,33 \%$ & $0,00 \%$ & $16,67 \%$ \\
Tetraciclina & $100,0 \%$ & $0,00 \%$ & $0,00 \%$ \\
\hline
\end{tabular}


De acordo com o estudo realizado por Freitas et al. (2005), que avaliou o perfil de sensibilidade antimicrobiana "in vitro" de Staphylococcus coagulase positivo isolados de leite de vacas com mastite, os antibióticos mais eficazes foram à vancomicina com $100 \%$ de e a norfloxacina com $96 \%$ e os menos eficazes foram a penicilina com apenas $20 \%$ de sensibilidade e amoxicilina com $25 \%$.

$\mathrm{Na}$ Tabela 4 estão listadas as cepas bacterianas utilizadas no presente trabalho de acordo com o número de princípios ativos aos quais apresentaram resistência e /ou sensibilidade, ou seja, o seu impacto para saúde pública.

De acordo com a Tabela 4 é possível observar que a cepa de Klebsiella spp. destacase quanto ao número de antimicrobianos aos quais foram resistentes, sendo esta $>50 \%$ dos antimicrobianos testados. No entanto, observou-se multirresistência em 50\% dos isolados: Hafnia alvei, Pantoea spp. e Klebsiella spp.

Assim, as três cepas podem ser consideradas de grande importância para saúde pública, uma vez que, podem chegar ao organismo humano veiculadas pelo leite, caso este não receba o tratamento térmico adequado e vir a tornar-se um problema na eventualidade de ser o agente etiológico de alguma infecção.
Apesar das bactérias isoladas neste estudo não estarem frequentemente associadas a intoxicação a partir da ingestão de alimentos contaminados, as mesmas apresentaram uma ampla resistência aos antimicrobianos usados no controle de infecções em humanos, e se presentes em abundância na cadeia alimentar, podem ter um efeito grave nas futuras opções de tratamento para uma ampla gama de infecções causada por estes micro-organismos (OVERDEVEST et al., 2011).

A presença dos micro-organismos resistentes à antimicrobianos, encontrados no presente estudo, associada ao hábito de consumo de leite e/ou derivados crus, ressalta a importância dos produtos lácteos como fonte de contaminação por esses micro-organismos. É importante controlar a presença desses micro-organismos durante a ordenha, armazenamento, transporte e beneficiamento do leite através de boas práticas de higiene durante a ordenha nas propriedades rurais e de fabricação nas indústrias de laticínios. Além disso, órgãos de fiscalização e vigilância epidemiológica em saúde devem atuar continuamente para coibir o comércio de leite e derivados crus e conscientizar os consumidores e comerciantes sobre os riscos relacionados ao consumo desses produtos crus.

Tabela 4 - Número de princípios ativos para os quais as cepas isoladas do leite cru do Maranhão apresentaram resistência, sensibilidade ou foram intermediárias

\begin{tabular}{|c|c|c|c|}
\hline Bactérias & Sensível & Intermediária & Resistente \\
\hline Klebsiella spp. & 5 & 0 & 6 \\
\hline Hafnia alvei & 6 & 0 & 5 \\
\hline Pantoea spp. & 7 & 0 & 4 \\
\hline L. garvieae & 7 & 1 & 3 \\
\hline A. septicus & 8 & 2 & 1 \\
\hline S. epidermidis & 11 & 0 & 0 \\
\hline
\end{tabular}




\section{CONCLUSÃO}

Cepas amplamente isoladas e descritas como componentes da microbiota mesófila e psicrotrófica do leite foram consideradas multirresistentes, como Klebsiella spp., Hafnia alvei e Pantoea spp. Os princípios ativos mais eficientes para controle de microorganismos potencialmente patogênicos ao homem foram gentamicina e tetraciclina, com $100 \%$ de eficiência frente as cepas testadas neste estudo.

\section{REFERÊNCIAS}

ALMEIDA, L. P. et al. Antibiotic residues in milk of rural properties of Uberlândia MG region. Bioscience Journal, v. 19, n. 3, p. 83-87, 2003.

BAUER A.W. et al. Antibiotic susceptibility testing by a standardized single disk method. American Journal of Clinical Pathology, v. 45 , n. 4 , p. 493-496, 1966. DOI: $10.1093 /$ ajcp/45.4 ts. 493 .

BICUDO, E. L. et al. Nosocomial outbreak of Pantoea agglomerans in a pediatric urgent care center. Brazilian Journal of Infectious Diseases, v. 11, n. 2, p. 281-284, 2007. DOI: $10.1590 / \mathrm{S} 1413-86702007000200023$.

CANTAS, L. et al. A brief multi-disciplinary review on antimicrobial resistance in medicine and its linkage to the global environmental microbiota. Frontiers in Microbiology, v. 4, n. 96, p. 1-14, 2013. DOI: 10.3389/ fmicb.2013.00096.

CLSI (Clinical and Laboratory Standards Institute). Performance Standards for Antimicrobial Susceptibility Testing. Twenty-Fifth Informational Supplement. CLSI Document M100-S25. Wayne: Clinical and Laboratory Standards Institute. 2015.
Disponível em: https://www.facm.ucl. ac.be/intranet/CLSI/CLSI-2015-M100-S25. unlocked.pdf. Acesso em: 07 set 2018.

CLSI (Clinical and Laboratory Standards Institute). Methods for Dilution Antimicrobial Susceptibility Tests for Bacteria that Grow Aerobically. 2012. Disponível em: http://antimicrobianos.com.ar/ ATB/wp-content/uploads/2012/11/03-CLSIM07-A9-2012.pdf. Acesso em: 14 jul. 2018.

ESTRELA, T. S. Resistência antimicrobiana: enfoque multilateral e resposta brasileira. In: B R A S L, M INISTÉRIO D A SAÚDE, ASSESSORIA DE ASSUNTOS INTERNACIONAIS DE SAÚDE. Saúde e política externa: os 20 anos da Assessoria de Assuntos Internacionais de Saúde (19982018). Brasília: Ministério da Saúde, 2018. p. 307-327.

FRACALANZZA, S. A. P. Identificação, resistência a antimicrobianos e caracterização molecular de Enterococcus isolados de alimentos. 2007. 177 f. Tese (Doutorado em Vigilância Sanitária) Instituto Nacional de Controle de Qualidade em Saúde, Fundação Oswaldo Cruz, Rio de Janeiro, 2007.

FREITAS, M. F. L. et al. Perfil de sensibilidade antimicrobiana in vitro de Staphylococcus coagulase positivos isolados de leite de vacas com mastite no agreste do Estado de Pernambuco. Arquivo do Instituto Biológico, v. 72, n. 2, p. 171-177, 2005.

FOOD AND DRUG ADMINISTRATION (FDA). New Drugs at FDA: CDER's new molecular entities and new therapeutic biological products. Disponível em: http://wayback.archive-it. org/7993/20170111002417/http://www.fda. gov/Drugs/DevelopmentApprovalProcess/ 
DrugInnovation/default.htm Acesso em: 31 jul. 2019.

GURUNG, M. et al. Prevalence and antimicrobial susceptibility of Acinetobacter from raw bulk tank milk in Korea. Journal of Dairy Science, v. 96, n. 4, p. 1997-2002, 2013.

JANDA, J. M.; ABBOTT, S. L. The genus Hafnia: from soup to nuts. Clinical Microbiology Reviews, v. 19, n. 1, p. 12-28, 2006. DOI: 10.1128/cmr.19.1.12-28.2006.

KILIC, A. et al. Acinetobacter septicus sp. nov. Association with a nosocomial outbreak of bacteremia in a neonatal intensive care unit. Journal of Clinical Microbiology, v. 46, n. 3, p. 902-908, 2008. DOI: 10.1128/ jcm. 01876-07

LIMA, J. B. A. Caracterização genética de bactérias proteolíticas e lipolíticas autóctones do leite cru no MaranhãoBrasil. 2016. 100 f. Tese (Doutorado em Ciência Animal) - Universidade Estadual de Londrina, Londrina, 2016.

MAGIORAKOS, A. P. et al. Multidrugresistant, extensively drug-resistant and pandrug-resistant bacteria: an international expert proposal for interim standard definitions for acquired resistance. Clinical Microbiology and Infection, v. 18, n.3, p. 268281. DOI:10.1111/j.1469-0691.2011.03570.x

OLIVEIRA, C. B. S. Frequência e perfil de resistência de Klebsiella spp. em um hospital universitário de Natal/RN durante 10 anos. Jornal Brasileiro de Patologia e Medicina Laboratorial, v. 47, n. 6, p. 589-594, 2011. DOI: $10.1590 / \mathrm{S} 1676-24442011000600003$.

OVERDEVEST, I. et al. Extended-spectrum $\beta$-Lactamase genes of Escherichia coli in chicken meat and humans, the Netherlands.
Emerging Infectious Diseases, v. 17, n. 7, p. 1216-1222, 2011.

PERNA, T. D. G. S. Prevalência de infecção hospitalar pela bactéria do gênero Klebsiella em uma Unidade de Terapia Intensiva. Revista da Sociedade Brasileira de Clínica, v. 13, n. 2, p. 119-123, 2015.

PIESSENS, V. et al. Intra-species diversity and epidemiology varies among coagulasenegative Staphylococcus species causing bovine intramammary infections. Veterinary Microbiology, v. 155, n. 1, p. 62-71, 2012. DOI:10.1016/j.vetmic.2011.08.005

PIERI, F. A. et al. Risky consumption habits and safety of fluid milk available in retail sales outlets in Viçosa, Minas Gerais State, Brazil. Foodborne Pathogens and Disease, v. 11, n. 6 , p. $490-496,2014$

POSSUElO, L. G.; RENNER, J. D. P. Caracterização e identificação microbiológica de Kruyvera sp. e Pantoea sp. Journal of Infection Control, v. 4, n. 2, p. 52-53, 2015.

ROCHA, C.; REYNOLDS, N. D.; SIMONS, M. P. Resistencia emergente a los antibióticos: una amenaza global y un problema crítico en el cuidado de la salud. Revista Peruana de Medicina Experimental y Salud Pública, v. 32, n. 1, p. 139-145, 2015.

SALABERRY, S. R. S. et al. Análise microbiológica e perfil de sensibilidade do Staphylococcus spp. em mastite subclínica de caprinos leiteiros. Arquivo Brasileiro de Medicina Veterinária e Zootecnia, v. 68, n. 2, p. 336-344, 2016.

SANTOS, N. Q. A resistência bacteriana no contexto da infecção hospitalar. Texto Contexto Enfermagem, v. 13, p. 64-70, 2004. SILVA, L. C. C.; FAGNANI, R. Resíduos 
químicos no leite. In: BELOTI, V. et al. Leite: obtenção, inspeção e qualidade. Londrina: Editora Planta, 2015.

TANWAR J. et al. Multidrug resistance: An emerging crisis. Interdisciplinary Perspectives on Infectious Diseases, n. 541340, p.1-7, 2014. DOI: $10.1155 / 2014 / 541340$.

TOMAZI, T. et al. Identification of coagulasenegative Staphylococci from bovine intramammary infection by Matrix-Assisted Laser Desorption Ionization-Time of Flight Mass Spectrometry. Journal of Clinical
Microbiology, v. 52, n. 5, p. 1658-1663, 2014. DOI: $10.1128 / \mathrm{jcm} .03032-13$.

VENDRELL, D. et al. Lactococcus garvieae in fish: A review. Comparative Immunology, Microbiology and Infectious Diseases, v. 29, n. 4, p. 177-198, 2006. DOI:10.1016/j.cimid.2006.06.003.

WILBRING, M. et al. Lactococcus garvieae causing zoonotic prosthetic valve endocarditis. Clinical Research in Cardiology, v. 100, n. 6, p. 545-546, 2011. DOI:10.1007/s00392-011-0286-3 